Volume 8, No.3, May - June 2019

International Journal of Advanced Trends in Computer Science and Engineering

Available Online at http://www.warse.org/IJATCSE/static/pdf/file/ijatcse77832019.pdf

https://doi.org/10.30534/ijatcse/2019/77832019

\title{
Next-Hour Electrical Load Forecasting using an Artificial Neural Network: Applicability in the Philippines
}

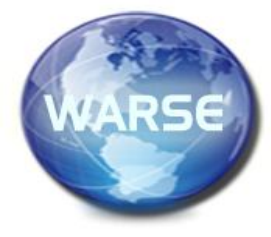

\author{
Lorwin Felimar Torrizo ${ }^{1}$, Aaron Don Africa ${ }^{2}$ \\ Department of Electronics and Communications Engineering \\ De La Salle University, Manila \\ 2401 Taft Ave., Malate, Manila 1004, \\ Philippines \\ ${ }^{1}$ lorwin_torrizo@dlsu.edu.ph, ${ }_{2}^{2}$ aaron.africa@dlsu.edu.ph
}

\begin{abstract}
Load forecasting remains as an important activity for the power systems industry, being a critical ystem. With the increasing amount of implemented microgrids across the Philippines, there is merit in investigating localized load forecasting schemes for use in assigning the dispatch of the microgrid's resource at any given time. This study reviews the use of an artificial neural network (ANN) as a next-hour load forecasting method in the Philippine setting.
\end{abstract}

Key words: Artificial Neural Networks, Load Forecasting, Philippines, Power Systems

\section{INTRODUCTION}

\subsection{Background}

It is a well-known fact that load forecasting, in the context of power systems, is a critical activity that aids those involved in the power sector towards meeting the demand requirements of the system. The demand for electricity changes within the day, and it is up to the utility companies to adjust their operation in order to meet their reliability requirements while maintaining economic operation. With proper forecasting, these companies can plan ahead to establish proper transition from one operating state to another.

Due to the importance of load forecasting, there have already been numerous studies and models, ranging from extrapolation and regression methods to fuzzy logic and neural network algorithms [1].

\subsection{Motivation and Objectives of the Study}

Partly due to the advancements in technology in utilizing renewable energy as a power source, there have been an increase in implementations of microgrids in the Philippines.
As of 2017, the amount of power supplied by off-grid power plants to the country is roughly around $465 \mathrm{MW}$, covering $2 \%$ of the country's total installed capacity [2].

As the electricity sources within a microgrid are operating in cooperation with one another (unlike in the major grid which is comprised by several entities that act as competitors to each other), there exists a balancing act on keeping the costs low across multiple sources while maintaining reliability of the supplied power to the consumers. This procedure of allocating resources within the microgrid, called the dispatch, relies on several pieces of information which includes projected loading conditions as well as environmental conditions based from the past and present situation.

It is possible to devise an algorithm that assists in optimizing the dispatch of a microgrid, but the algorithm itself would necessitate a load forecasting algorithm within its structure. Herein lies the objective of this study: to affirm the applicability of using ANN as a load forecasting method in the Philippine context, for its eventual use in optimizing a local microgrid's dispatch. While an earlier study had already provided a short-term, neural network-based demand forecasting using PALECO load data [3], this study has adjustments in its selected input variables, as well as utilizing a different data set.

\section{RELATED LITERATURE}

There are a multitude of studies that attempt to utilize artificial neural networks as a tool in forecasting both short and long term load, even ranging as far back as nearly three decades ago.

\subsection{Previous load forecasting studies}

Earlier studies consider historical loading conditions combined with temperature as the predictors of hourly or daily load [4]-[8], with several studies referencing that utilizing wind speed and humidity might lead to higher accuracies [6], [9]. Reference [10] even attempted to 
differentiate between weekdays, weekends, and holidays its training data.

Recent studies maintain this trend of utilizing load and temperatures as input data [11], [12], with some variations like [13] which introduces sporadically spaced historical load for additional trend-finding, [14] which removes temperature considerations entirely while focusing on predicting through time and day of the week, and [15] which disregards historical load data and focuses on predicting through temperature and humidity alone. Reference [16] even considers natural gas and water consumption data.

\subsection{Load forecasting studies with Philippine context}

The study by Velasco, Palahang, Villezas, and Dagaang utilized raw monthly electric data from 2012 to 2014, accompanied by several time related variables (month, day, day of the week, week number, and weekend indicator) in order to predict the next-day hourly data [17].

The work by Bantugon and Gallano provided two load forecasting algorithms, one for short term forecasting (hourly) and the other for yearly, long term forecasting. Their hourly model utilized historical hourly load, minimum and maximum temperature, precipitation, and if it was a weekday or weekend; on the other hand, the yearly model only considered aggregated annual peak load, the annual gross domestic product (GDP), and the country's population [3].

The group of Atienza, Jao, Angeles, and Singzon also did a study in predicting regional electricity consumption, although the study primarily focused on comparing traditional back propagation ANN to that utilizing particle swarm optimization. Their focus was on predicting yearly demand, and the inputs utilized were electrical consumption, population, temperature, GDP, and employment rate [18].

\section{METHODOLOGY}

\subsection{Data gathering}

One of the recurring issues in most of the studies listed in the related literature is the lack of data available publicly. For the purposes of this study, however, publicly available sources of hourly electrical load and daily meteorological data exist.

\subsubsection{Meteorological Data}

In obtaining the necessary climate-related data, a reputable database of worldwide meteorological information should be utilized. For several programs that assist in designing renewable energy related projects, there are two well-known resources that openly provide historical information of several solar and wind energy parameters: the National Aeronautics and Space Administration (NASA) [19] and the National Renewable Energy Laboratory (NREL) [20].

NASA's POWER project is, according in its website, "initiated to improve upon the current renewable energy data set" and obtains its data from current satellite systems. Through its Data Access Viewer [21], with its GUI shown in Figure 1, a user is able to collect several meteorology parameters such as moisture, temperature, wind, and solar irradiance once latitude and longitude of the location has been specified. For this study, obtaining the daily air temperature values will assist in predicting the hourly load conditions.

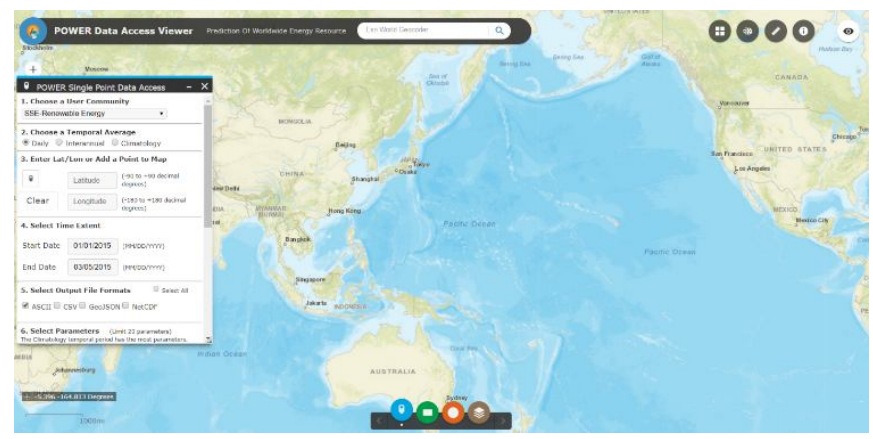

Figure 1: NASA's POWER Data Access Viewer

One of the limitations of NASA's POWER project is the unavailability of hourly data. While NASA's POWER is capable of providing high, low, and average temperatures for the day, these daily averages are insufficient for an algorithm that predicts hourly load conditions. In this scenario, the study utilizes an algorithm that determines the hourly temperature based from the maximum and minimum temperature and the time of sunrise [22]. This leads to obtaining the sunrise times, which can be provided by NREL's Solar Position and Intensity (SOLPOS) [23]. By specifying the location and the time frame, a user can utilize the SOLPOS algorithm using the provided $\mathrm{C}$ code or through an online calculator that can output a compressed file containing the requested solar parameters [24]. The SOLPOS calculator is shown in Figure 2.
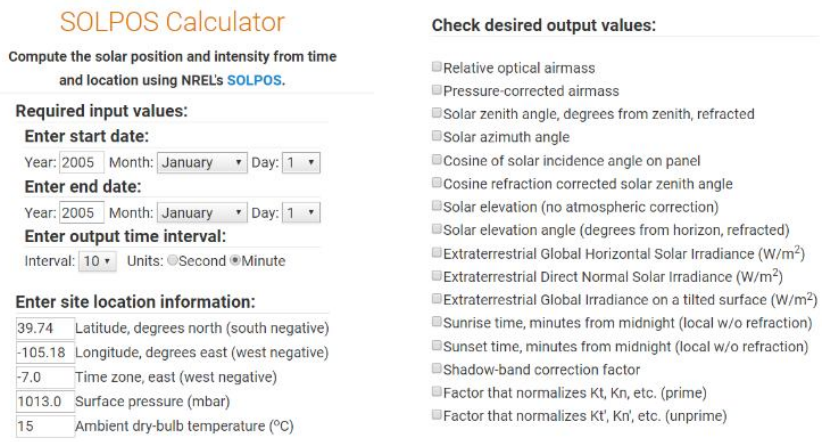

Figure 2: NREL's online SOLPOS calculator

\subsubsection{Hourly Load Profile}

Obtaining localized hourly load profile from any power system involves obtaining sensitive information and is thus met with high difficulty. In order to bypass the difficulty of obtaining permission for load profiles, this study instead opts for publicly available load profile data. Fortunately, the National Grid Corporation of the Philippines (NGCP) openly 
provides several operations data such as system peak demand, energy delivery per region, and hourly demand starting from 2012 which includes localized information for the major grids of the country's three island groups [25]. Their portal is as shown in Figure 3.
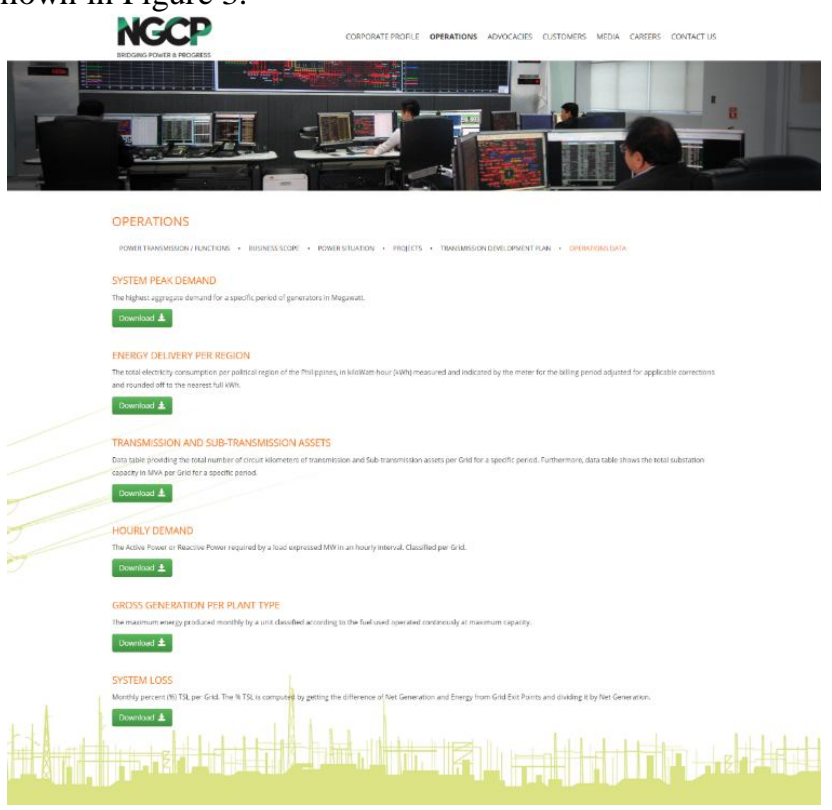

Figure 3: NGCP's portal for operations data

As both POWER and SOLPOS require a geographical location in order to provide their meteorological data, the coordinates of the country's capital, Manila $\left(14.59^{\circ} \mathrm{N}\right.$, $120.98^{\circ} \mathrm{E}$ ) will be used. It also follows that the study use the Luzon Hourly Data obtained from NGCP as there is where the capital is located.

\subsubsection{Summarizing the Data Set}

Figure 4 lists the corresponding input and output variables of the devised artificial neural network.

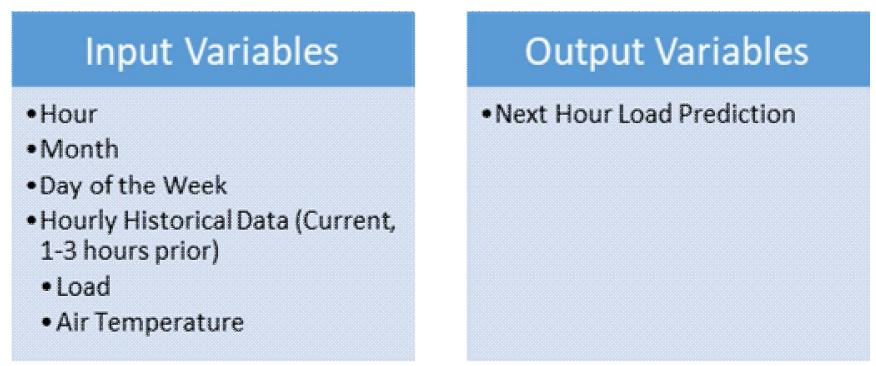

Figure 4: The devised ANN's input and output

The time-related inputs will be one hot encoded to fit the training process. On the other hand, it is necessary to normalize magnitude-based inputs prior to training and testing in order to reduce training time as well as avoid unnecessary errors. The minmax function is used in this study to lock the values between zero and one.

$$
\text { Minmax Normalization: } f(x)=\frac{x-\min (x)}{\max (x)-\min (x)}
$$

This study will utilize four years of information (January 2012 to December 2015) containing both its training and testing data. Around 35,000 data points were utilized in training and testing the derived artificial neural network, with $75 \%$ of the data to be used for training.

Lastly, the artificial neural network is comprised of at most 50 input nodes (majority of which are representing hour and month) and one output node (the forecasted load). A single hidden layer will complete its entire architecture.

\subsection{Code}

Majority of the code is based from Edureka's implementation of a Naval Mine Identifier using Deep Neural Networks [26], which was designed to identify whether the object detected underwater through sonar information was either a rock or an explosive mine. The implementation of the code, written using Python [27], utilizes several libraries that assists in processing and presenting the data within:

- TensorFlow [28]: provides several deep learning functions, including backpropagation mechanisms

- NumPy [29]: provides several array processing functions

- pandas [30]: used for importing .csv files

- sklearn [31]: provides additional array processing functions for shuffling and segmenting the data

As Edureka's implementation is a classifier, the code is modified to be used as a regressor, removing the softmax function and instead utilizing mean square error (MSE) as the cost function for training the ANN.

\section{RESULTS AND DISCUSSION}

Two data sets were used in the process of deriving a fitting artificial neural network: one including hour-related nodes as part of the input variables, and one without. The corresponding MSEs for both scenarios are illustrated in Figure 5 and tabulated in Table 1 below.

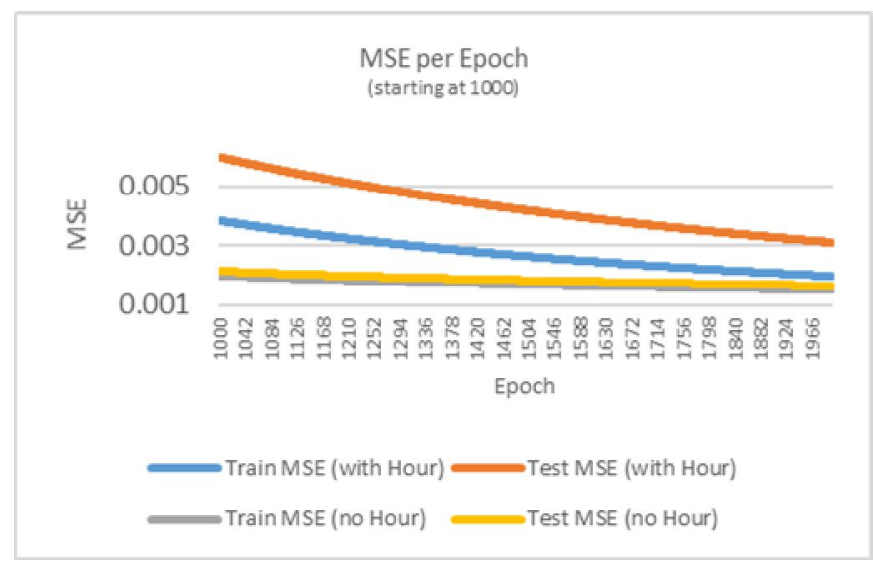

Figure 5: MSE per epoch with normalized output 
Table 1. Final MSE after 2000 epochs

\begin{tabular}{|c|c|c|c|}
\hline \multirow{2}{*}{ After 2000 epochs } & \multicolumn{2}{|c|}{ M SE } & \multirow{2}{*}{$\%$ diff } \\
\cline { 2 - 3 } & Training & Testing & \\
\hline With Hour input & 0.00196 & 0.00310 & $58.27 \%$ \\
\hline Without Hour input & 0.00150 & 0.00163 & $8.52 \%$ \\
\hline
\end{tabular}

Even after disregarding the higher MSE of the derived ANN that includes hour inputs, the relatively high difference between its training and testing MSE indicates that including hour input in the data sets leads to slight overfitting. Moving forward, the dataset with no hour-related inputs were used.

In order to evaluate the accuracy, the output minmax values were converted back to actual megawatt amount, and then the results of the test data are then compared against actual data using Mean Absolute Percentage Error (MAPE).

$$
M A P E=\frac{1}{n} \sum_{t=1}^{n}\left|\frac{A_{t}-\bar{F}_{t}}{A_{t}}\right| \times 100 \%
$$

The evaluation of the actual values and MAPE of the predicted and actual loads is illustrated by Figures 6 and 7 .

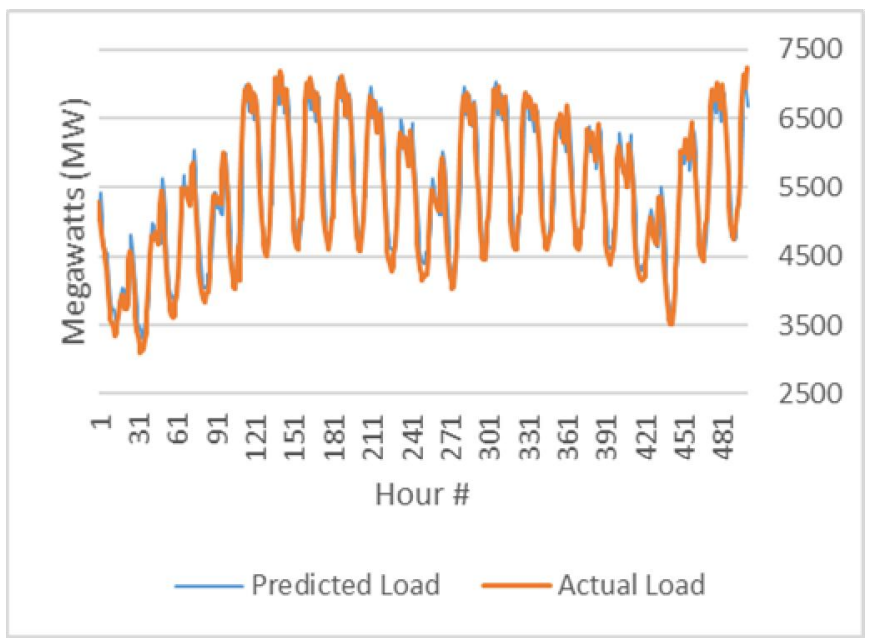

Figure 6: Predicted vs Actual Load (first 500 test points)

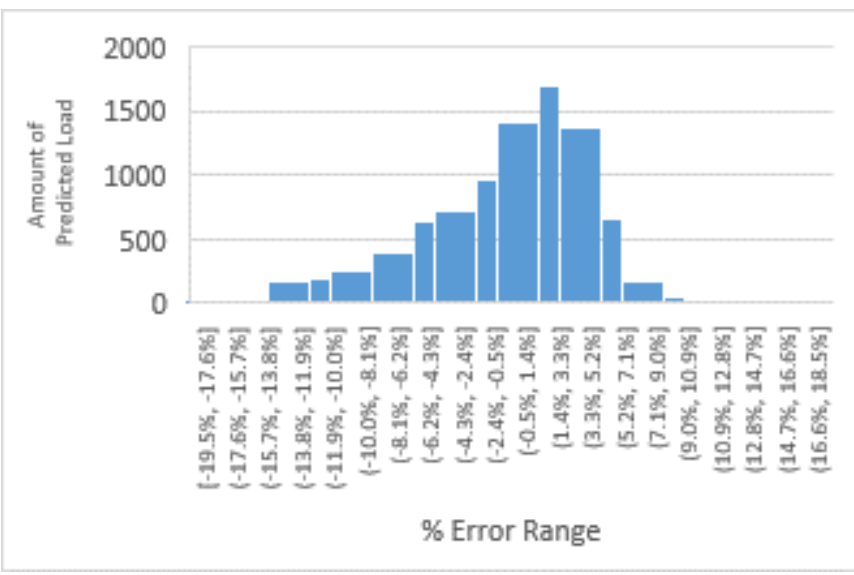

Figure 7. Distribution of percent error
Once converted to actual values, it can be seen that majority of the percent errors range from $-0.5 \%$ to $5.2 \%$. When evaluating the error using all the 8766 predicted hourly loads, the mean absolute percent error is $3.85 \%$.

\section{CONCLUSION}

Load forecasting utilizing artificial neural networks has already been established by multiple studies in the past. Nevertheless, this study investigates the applicability of ANN load prediction for the Philippines by utilizing publicly available data.

It is particularly hard to evaluate how 'good' the acquired MAPE of $3.85 \%$ is. Previous studies have reported errors as low as sub- $1 \%$ and as high as $8 \%$, in which was still concluded as 'very good in terms of MAPE'. Since the motivation of this study stems from utilizing load forecasting for the dispatch algorithm of a microgrid, it can be argued that as long as the error of prediction is low enough, the algorithm can work around the inaccuracy of its predicted load and maintain optimal dispatch. If accuracy remains a concern, there is still room for improvement by clearing the dataset of any outliers, differentiating a holiday, or just simply training the ANN further. Regardless, the relative closeness of MSEs between training and testing data brings confidence to the applicability of the ANN model that this study has presented.

\section{REFERENCES}

[1] L. Suganthi and A. A. Samuel, "Energy models for demand forecasting - A review," Renew. Sustain. Energy Rev., vol. 16, no. 2, pp. 1223-1240, 2012. https://doi.org/10.1016/j.rser.2011.08.014

[2] DOE-EPIMB, "2017 Power Demand and Supply Highlights," 2018.

[3] M. J. T. Bantugon and R. J. C. Gallano, "Short-and long-term electricity load forecasting using classical and neural network based approach: A case study for the Philippines," IEEE Reg. 10 Annu. Int. Conf. Proceedings/TENCON, pp. 3822-3825, 2017. https://doi.org/10.1109/TENCON.2016.7848779

[4] D. C. Park, M. A. El-Sharkawi, R. J. Marks, L. E. Atlas, and M. J. Damborg, "Electric Load Forecasting Using An Artificial Neural Network," IEEE Trans. Power Syst., vol. 6, no. 2, pp. 442-449, 1991. https://doi.org/10.1109/59.76685

[5] D. Srinivasan, A. C. Liew, and J. S. P. Chen, "A novel approach to electrical load forecasting based on a neural network," [Proceedings] 1991 IEEE Int. Jt. Conf. Neural Networks, pp. 1172-1177, 1991.

[6] M. A. El-Sharkawi, S. Oh, R. J. Marks, M. J. Damborg, and C. M. Brace, "Short term electric load forecasting using an adaptively trained layered perceptron," Proc. First Int. Forum Appl. Neural Networks to Power Syst., pp. 3-6, 1991.

[7] D. C. Park, M. Osama, M. A. El-Sharkawi, and R. J. Marks, "Adaptively trained neural networks and their 
application to electric load forecasting," IEEE Int. Symp. Circuits Syst., no. 5, pp. 1125-1128, 1991.

[8] S. J. Kiartzis, A. G. Bakirtzis, and V. Petridis, "Short-term load forecasting using neural networks," Electr. Power Syst. Res., vol. 33, no. 1, pp. 1-6, 1995. https://doi.org/10.1016/0378-7796(95)00920-D

D. C. Park and O. Mohammed, "Artificial neural network based electric peak load forecasting," IEEE Proc. SOUTHEASTCON '91, pp. 225-228, 1991.

[10] D. Srinivasan, A. C. Liew, and J. S. P. Chen, "Short term forecasting using neural network approach," Proc. First Int. Forum Appl. Neural Networks to Power Syst., pp. 12-16, 1991.

[11] A. Webberley and D. W. Gao, "Study of Artificial Neural Network Based Short Term Load Forecasting," pp. 7-10, 2013. https://doi.org/10.1109/PESMG.2013.6673036

[12] W. He, "Load Forecasting via Deep Neural Networks," Procedia Comput. Sci., vol. 122, pp. 308-314, 2017.

https://doi.org/10.1016/j.procs.2017.11.374

[13] S. Singh, "Short term load forecasting using artificial neural network," pp. 159-163, 2017. https://doi.org/10.1109/ICIIP.2017.8313703

[14] L. Zhuang, H. Liu, J. Zhu, S. Wang, and Y. Song, "Comparison of forecasting methods for power system short-Term load forecasting based on neural networks," 2016 IEEE Int. Conf. Inf. Autom. IEEE ICIA 2016, no. 61473174, pp. 114-119, 2017. https://doi.org/10.1109/ICInfA.2016.7831806

[15] M. K. Singla, J. Gupta, and P. Nijhawan, "Comparative Study on Backpropagation and Levenberg Marquardt Algorithm on Short Term Load Forecasting," Int. J. Adv. Trends Comput. Sci. Eng., vol. 8, no. 2, pp. 194-202, 2019.

https://doi.org/10.30534/ijatcse/2019/14822019

[16] T. Hossen, A. S. Nair, R. A. Chinnathambi, and P. Ranganathan, "Residential Load Forecasting Using Deep Neural Networks (DNN)," 2018 North Am. Power Symp. NAPS 2018, pp. 1-5, 2019. https://doi.org/10.1109/NAPS.2018.8600549

[17] L. C. P. Velasco, C. R. Villezas, P. N. C. Palahang, and J. A. A. Dagaang, "Next day electric load forecasting using Artificial Neural Networks," no. December, pp. 1-6, 2016.

https://doi.org/10.1109/HNICEM.2015.7393166

[18] N. A. C. Atienza, J. R. A. T. Jao, J. A. D. S. Angeles, E. L. T. Singzon, and D. D. Acula, "Prediction and Visualization of Electricity Consumption in the Philippines Using Artificial Neural Networks, Particle Swarm Optimization, and Autoregressive Integrated Moving Average," 2018 3rd Int. Conf. Comput. Commun. Syst. ICCCS 2018, pp. 135-138, 2018.

https://doi.org/10.1109/CCOMS.2018.8463351

[19] "NASA." [Online]. Available: https://www.nasa.gov/. [Accessed: 03-May-2019].

[20] "National Renewable Energy Laboratory (NREL) Home Page | NREL." [Online]. Available: https://www.nrel.gov/. [Accessed: 03-May-2019].
[21] "POWER Data Access Viewer." [Online]. Available: https://power.larc.nasa.gov/data-access-viewer/.

[Accessed: 04-May-2019].

[22] D. H. C. Chow and G. J. Levermore, "New algorithm for generating hourly temperature values using daily maximum, minimum and average values from climate models," no. August, 2007.

https://doi.org/10.1177/0143624407078642

[23] "Solar Position and Intensity | Grid Modernization | NREL." [Online]. Available: https://www.nrel.gov/grid/solar-resource/solpos.html . [Accessed: 05-May-2019].

[24] "SOLPOS Calculator." [Online]. Available: https://midcdmz.nrel.gov/solpos/solpos.html.

[Accessed: 05-May-2019].

[25] "Operations - National Grid Corporation of the Philippines." [Online]. Available: https://www.ngcp.ph/operations\#operations.

[Accessed: 05-May-2019].

[26] "edureka! - YouTube." [Online]. Available: https://www.youtube.com/user/edurekaIN.

[Accessed: 11-Aug-2018].

[27] “About Python ${ }^{\mathrm{TM}}$ | Python.org." [Online]. Available: https://www.python.org/about/. [Accessed: 20-Jun-2019].

[28] "TensorFlow." [Online]. Available: https://www.tensorflow.org/about. [Accessed: 20-Jun-2019].

[29] "NumPy — NumPy." [Online]. Available: https://www.numpy.org/. [Accessed: 20-Jun-2019].

[30] "The pandas project — pandas: Python Data Analysis Library." [Online]. Available: https://pandas.pydata.org/about.html. [Accessed: 20-Jun-2019].

[31] "scikit-learn: machine learning in Python scikit-learn 0.21.2 documentation." [Online]. Available: https://scikit-learn.org/stable/. [Accessed: 20-Jun-2019]. 